\title{
Numerical Study of Electromagnetic Interaction between a Radio Frequency Sources and the Human Head
}

\author{
Bouali Lazhar, Djennah Mohammed \\ Laboratory of Electrotechnic - enp - Algeria
}

\begin{abstract}
In This part of our work, we present a model based on a numerical calculation to solve the problem of the effects of radio frequency electromagnetic radiation on human ORL sphere and select a good protector against the electromagnetic field that is the human head-mobile interface. In other words, it is the effect of the use of mobile on human tissue. Indeed, it is an electromagnetic coupling between a source of electromagnetic energy and the human body. To solve the system of equations governing this problem, a finite element formulation coupled with boundary integral formulation that is adopted. The numerical results obtained with this method show the influence of characteristic parameters, $(\varepsilon, \sigma, \mu)$ of exterior medium on penetration electromagnetic field in human head. There inside the head we can evaluate electromagnetic energy at each point inside the human head sphere functioned characteristics parameters of exterior medium (the interface mobile- human head).
\end{abstract}

Keywords: head, cellular, field

\section{Introduction}

The development of communications without lines is huge; it reaches an advanced point on the scale of progress. This phenomenon contributes to the expansion in our society many electromagnetic waves based systems (mobile phone base station microwave ovens...). These have various biological effects such as tissue temperature rise, and other, which carries most of the current research.

The objective of our study is to reach the ability to select aninterface mobile- human head by the use of numerical modeling of electromagnetic interaction between these two last elements. This allows energy assessment of electromagnetic fields in different parts of the human head model with worn as the variation of parameters $(\sigma, \varepsilon, \mu)$ interface mobile- human head, hence the electromagnetic field values are important and gradually decrease rapidly as one moves away from the antenna of the radio and that it approaches the center of the head.

In our study, we are interested in the electromagnetic field radiated. figure.01. It shows a configuration of a model problem related to bio- electromagnetic compatibility, or the human head is spherical radius $r$ containing the brain is considered to be homogeneous and isotropic, identified an external environment radius. Our sphere illuminated by electromagnetic radiation from a source located in the outer region, considered harmonic in time.

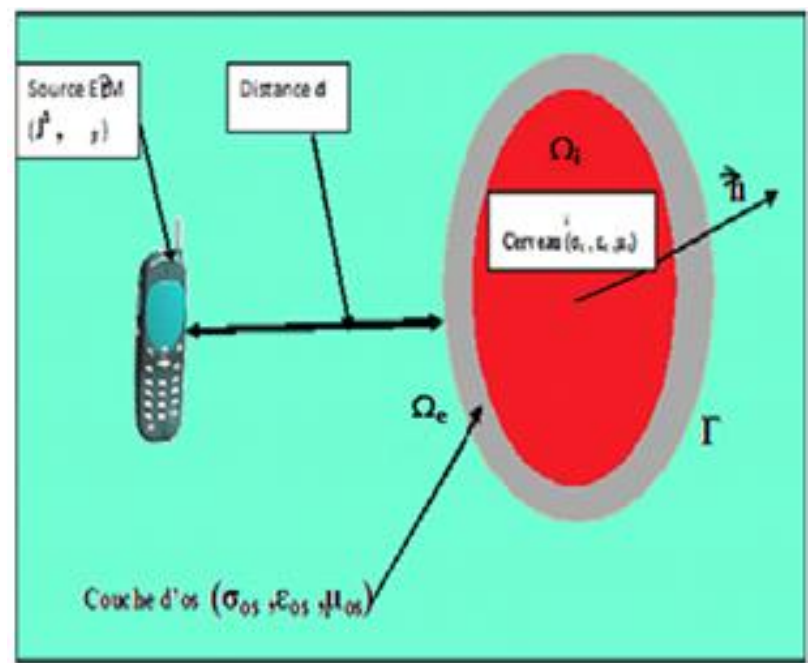

Figure 1: Physical system

In our study, we first apply the finite element method inside and on the head. We express the external environment by an integral representation. Itis based on the equivalent electric current $\mathrm{K}$ carried by the outer surface of the human head. These surface currents represent the jump of the tangential component of the magnetic field:

$$
[n \times h]_{\Gamma}=K
$$

We realize the coupling of the two circles by calculation of integral-differential operator linking the traces of the tangential component of the electric field $\mathrm{R}$ and that of its rotation [5]:

$$
R(n \times e)=n \times \operatorname{curl}(e)
$$

This term represents the period of the border in the variational formulation of the problem. We calculate the operator $\mathrm{R}$ using $\mathrm{K}$ as an intermediary unknown factor between the inner and outer problem.

\section{Formulation}

The physical system defined by the following equations: 
- In the head:

$$
\begin{array}{r}
i \omega \mu_{0} h+\operatorname{curl}(e)=0 \\
-i \omega \varepsilon_{0} e+\operatorname{curl}(h)=0
\end{array}
$$

- In the medium without external source:

$$
\begin{aligned}
& i \omega \mu_{0} h+\operatorname{curl}(e)=0 \\
& -i \omega \varepsilon_{0} e+\operatorname{curl}(h)=0
\end{aligned}
$$

Inaddition, we take in any system:

$$
\operatorname{div} \mu h=0 ; \operatorname{div} \varepsilon e=0
$$

With these equations, we take into account the conditions at infinity:

$$
\begin{gathered}
e=0(1 / r) \\
h=0(1 / r)
\end{gathered}
$$

We take as constant scalar functions in each different region, where $\mathbf{h}$ and $\mathbf{e}$ are the magnetic and electric fields respectively; $\sigma$ is the electrical conductivity and the electrical permittivity $\varepsilon, \boldsymbol{\mu}$ and magnetic permeability.

\section{The Variational Problem}

In order to evaluate the electromagnetic field inside the human head and we formulate our problem in the electrical field:

$$
\frac{1}{\mu} \operatorname{curl} \operatorname{curl}(e)+i \omega(\sigma+i \omega \varepsilon) e=0
$$

Multiplying equation (12) by a test function of e 'and integrate the volume of the domain occupied by the $\Omega p$ human head, we have

$$
\begin{aligned}
& \int_{\Omega} \operatorname{curl}(e) \cdot \operatorname{curl}\left(e^{\prime}\right) d \Omega+\int_{\Gamma}(n \cdot \operatorname{curl}(e)) e^{\prime} d \Gamma \\
& +i \omega \mu(i \omega \varepsilon+\sigma) \int_{\Omega} e \cdot e^{\prime} d \Omega=0
\end{aligned}
$$

$\Gamma$ The border of $\Omega$ and $\mathrm{n}$ is the outward unit normal to $\Gamma$. The total electric field $\mathrm{E}$ is theoretically composed of two parts, one of the first reaction and the other of the source es

$$
e=e^{s}+e^{r}
$$

With this decomposition, we can write (12) as an integral equation where the unknown is er in the first term and the second member is a function of es...

\section{Calculation the Border Term}

ГIn the integral equation (12) called border of term. The integral term on the other hand, the solution of the internal problem could not be the solution in de $\Gamma$ this point it should be noted on the first hand that we cannot solve the problem without identifying the jump the tangential component of the magnetic field on the problem in the open space R3. Only the term Tborder allows us to take into account the behavior of the field in the external environment. To treat the term of border $\Gamma$, we will first formulate the problem and express its outdoor solution for traces of the border field. The system of equations (6) - (9) controls the behavior of the magnetic field in the external environment. The solution of the problem in the environment can written in the following integral form:

$$
e(x)=\mu_{0} \int_{\Gamma} K(y) \cdot G(x, y) d y
$$

Where $\mathrm{K}$ is the jump of the tangential component of the magnetic field:

$$
[n \wedge h]=K
$$

The current equivalent area $\mathrm{K}$ filed on $\Gamma$ is fictional.

$e_{0}=n \times e(x)=\mu_{0} \cdot \int_{\Gamma} n \times K(y) \cdot G(x, y) d y$

$\left\langle e^{\prime}, K^{\prime}\right\rangle=\left\langle e_{0}, K^{\prime}\right\rangle=\frac{1}{4 \pi} \int_{\zeta} \int_{\Gamma} \frac{K(y) \cdot K^{\prime}(x)}{|x-y|} d x d y$

It also known as an intermediate used to couple the internal and external problems. We will have $\mathrm{K}$ expressed as e. To keep the electric field $\mathrm{E}$ as the only unknown actor on $\Gamma$

$$
[n \wedge h]=R(e) \text {. }
$$

Our goal now is to calculate $\mathrm{R}$. This calculation based on a variational formulation:

\section{Numerical Implementation}

Numerically solve the problem, the volume of study split into tetrahedral elements and the electric field vector $\mathrm{E}$ described in terms of basic functions Wij associated with the edges of these elements.

$$
\begin{gathered}
e=\sum_{i=1}^{N} W_{i j} \cdot e_{i} \\
W_{i j}=\lambda_{a} \nabla \lambda_{b}-\lambda_{b} \nabla \lambda_{a}
\end{gathered}
$$

Where $\mathrm{N}$ is the total number of edges of the mesh, Wij is the basic function of the vector associated with edge (ij) and e ij is the unknown problem that represents the flow of electric field along the edge (ij). $i$ is the barycentric coordinate i. $\lambda$.Tetrahedral associated with the node.

We have developed the $\mathrm{K}$ currents on the basis function:

$$
\omega_{i}(x)=n(x) \times \operatorname{grad} \lambda_{i}
$$

And

$$
K(x)=\sum_{i} P_{i} \cdot \omega_{i}(x)
$$

Where:

. $\mathrm{i}$ : Describes the top

$\mathrm{Pi}$ : the value of $\mathrm{K}$ in vertex $\mathrm{i}$.

$\mathrm{i}$ : the coordinated barycentric. $\lambda$

. . $\mathrm{\Gamma n}(\mathrm{X})$ : normal vector on

Our variational formulation can rewritten as the following linear forms:

$$
\begin{aligned}
& M_{G R} \cdot e_{V}=S_{V} \\
& M_{G C} \cdot e_{C}=S_{C}
\end{aligned}
$$

Where:

$M_{G V}=M_{1}+R+j \omega \sigma \mu \cdot M_{2}$

$M_{G C}=M_{1}+R$ 


\section{International Journal of Science and Research (IJSR) \\ ISSN (Online): 2319-7064}

Index Copernicus Value (2013): 6.14 | Impact Factor (2014): 5.611

A: is a complete matrix of dimension

(Nbat $\times$ nbat); it represents the boundary term of the approximate variational problem.

M1 and M2. Are two matrices; their dimensions are

(nbat $\times$ nbat); an element of these matrices is equal to zero only if (ij) and (kl) are not part of the same tetrahedron.

\section{Numerical Results}

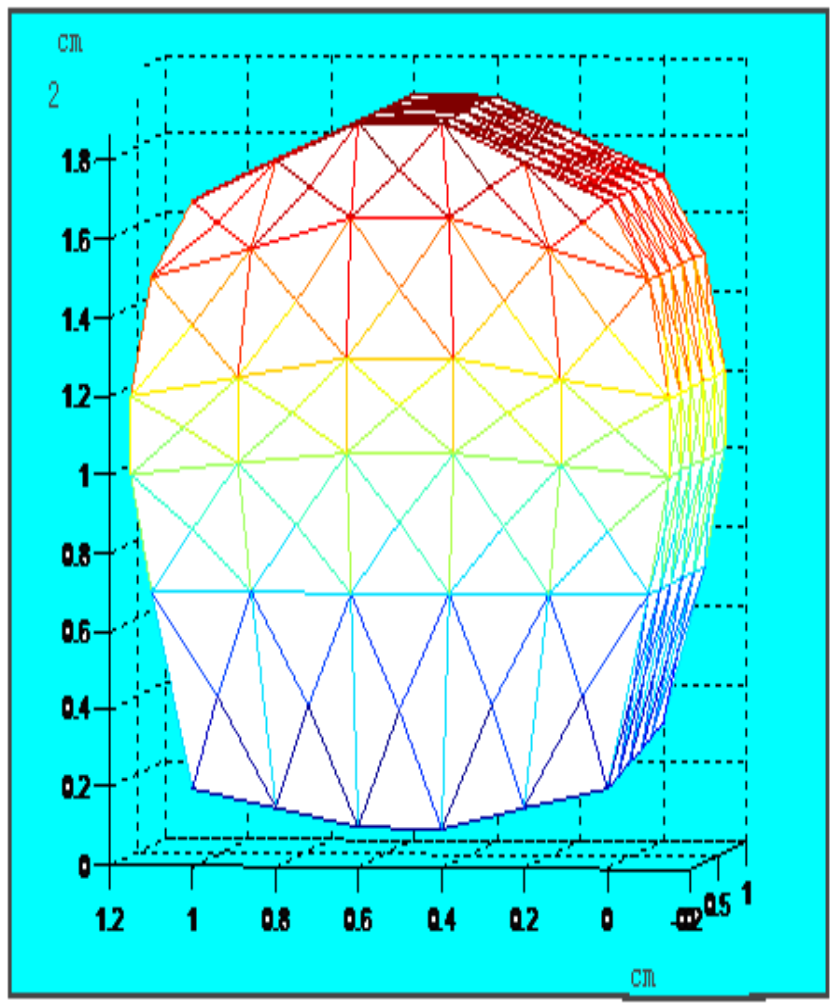

Figure 2: Representation of a grid mesh

Number of internal vertices 12025

Number of border summits 2946

Number of internal edges 73594

Number of border edges 8832

Number of facets 131968

Number of Border facets 5888

Number of tetrahedral 64512

Number of tetrahedral tangent 21280

Number of Internal tetrahedral 43232

\section{Representation of Field}

With

$\mathrm{JS}=100, \mathrm{R}=0,2 \mathrm{VN} 1=-1, \mathrm{VN} 2=0,=0 \mathrm{VN} 3$

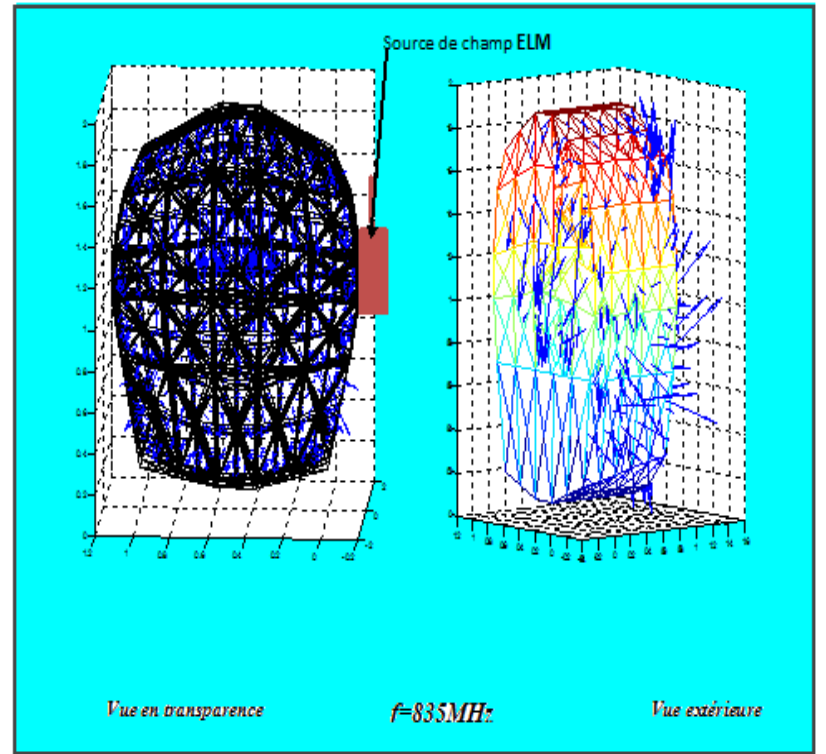

Figure 3: Distribution of the electric field Source glued to the head $\mathrm{f}=835 \mathrm{MHz}$

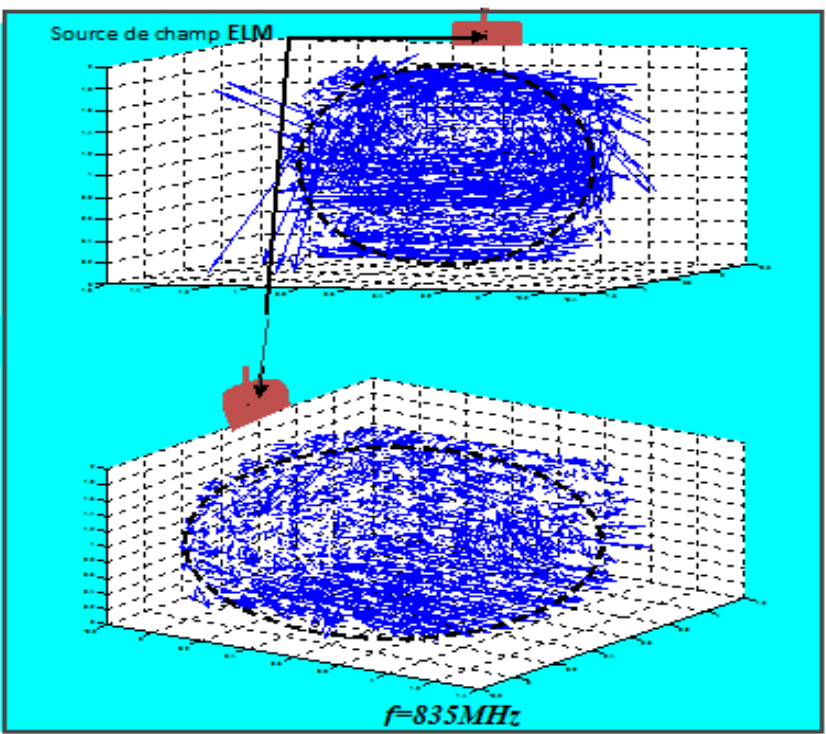

Figure 4: Distribution of the electric field $\mathrm{E}$ Source glued to the head $\mathrm{f}=835 \mathrm{MH}$ 
International Journal of Science and Research (IJSR)

ISSN (Online): 2319-7064

Index Copernicus Value (2013): 6.14 | Impact Factor (2014): 5.611

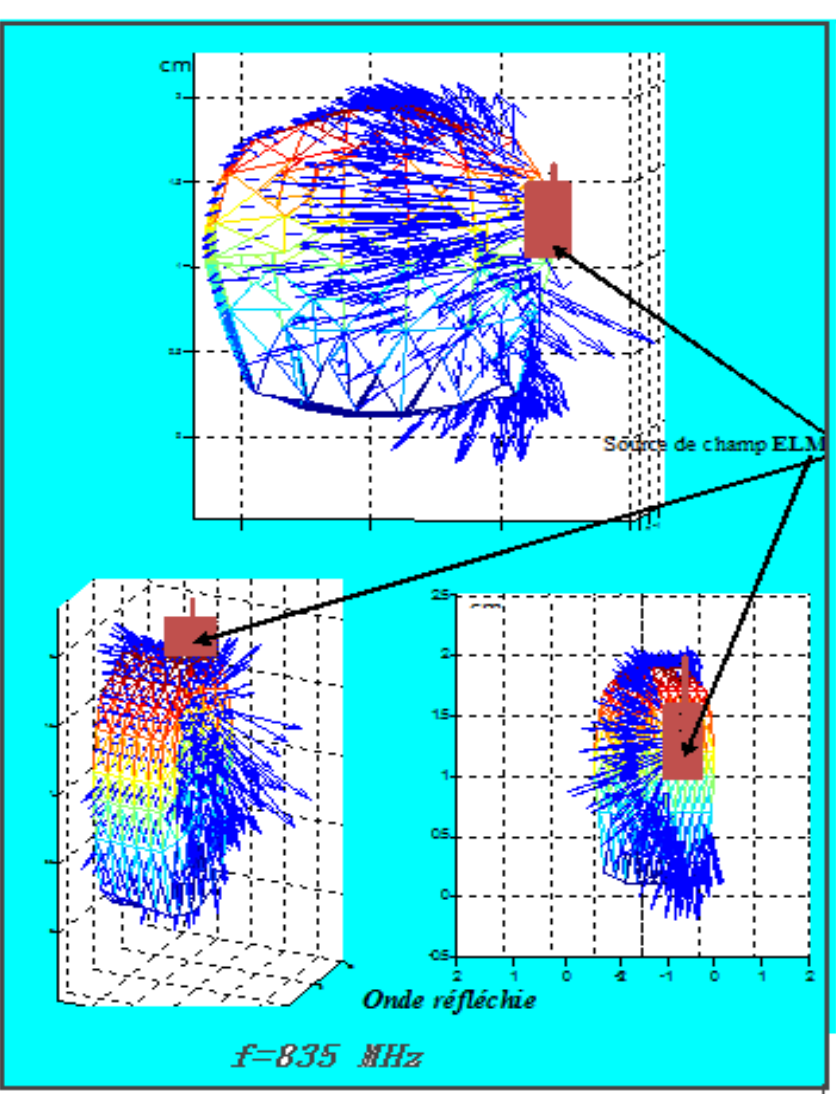

Figure 5: Distribution of Magnetic Field Source glued to the head $\mathrm{f}=835 \mathrm{MHz}$

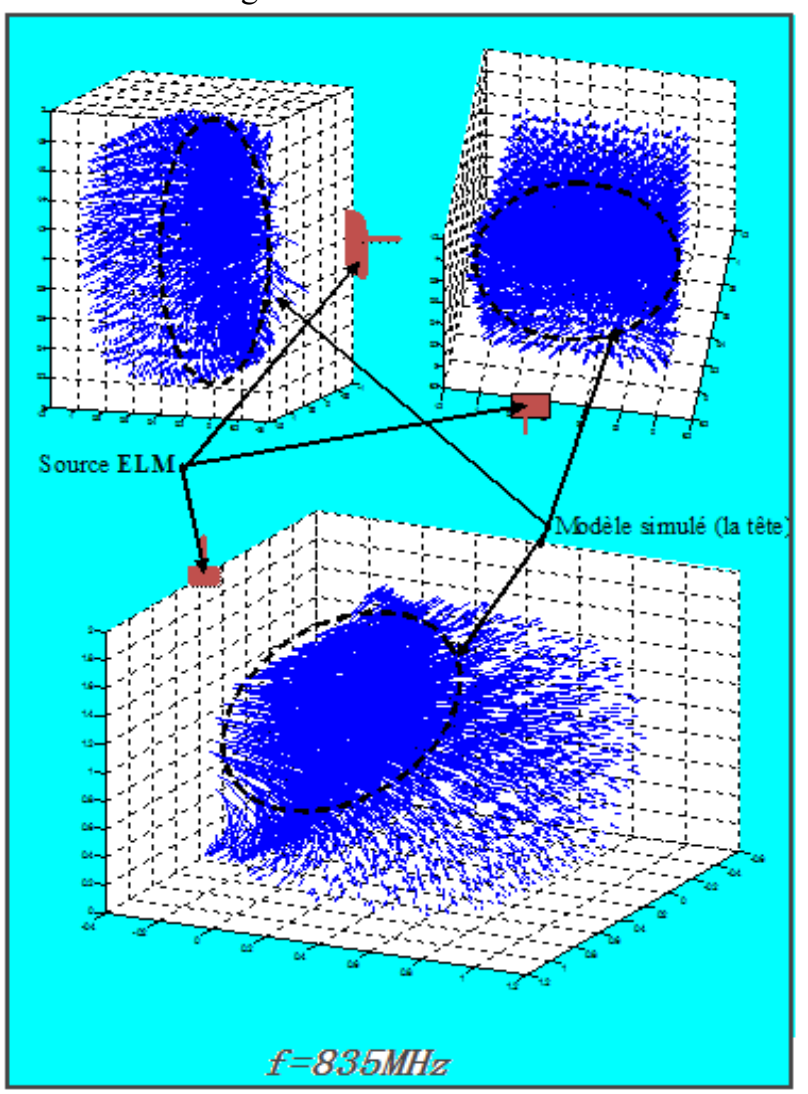

Figure 6: Distribution of the magnetic field $\mathrm{H}$ Source glued to the head $\mathrm{f}=835 \mathrm{MHz}$

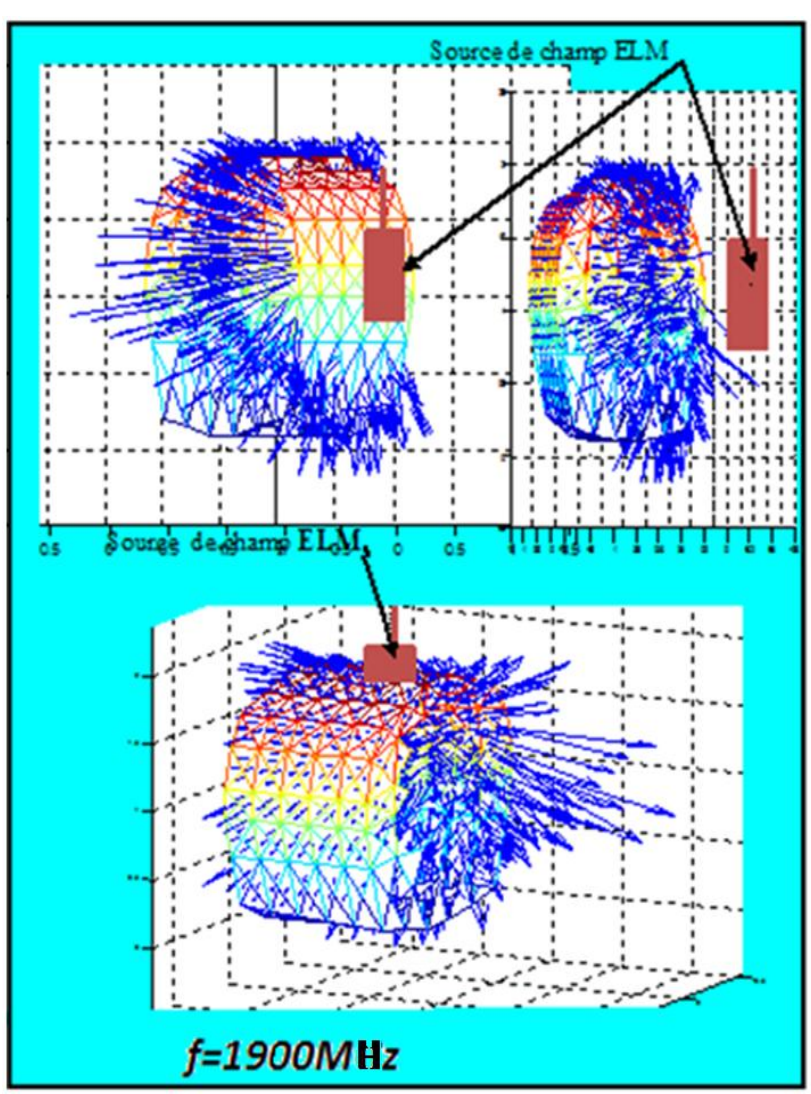

Figure 7: Distribution of the magnetic field Source glued to the head $\mathrm{f}=1900 \mathrm{MHz}$

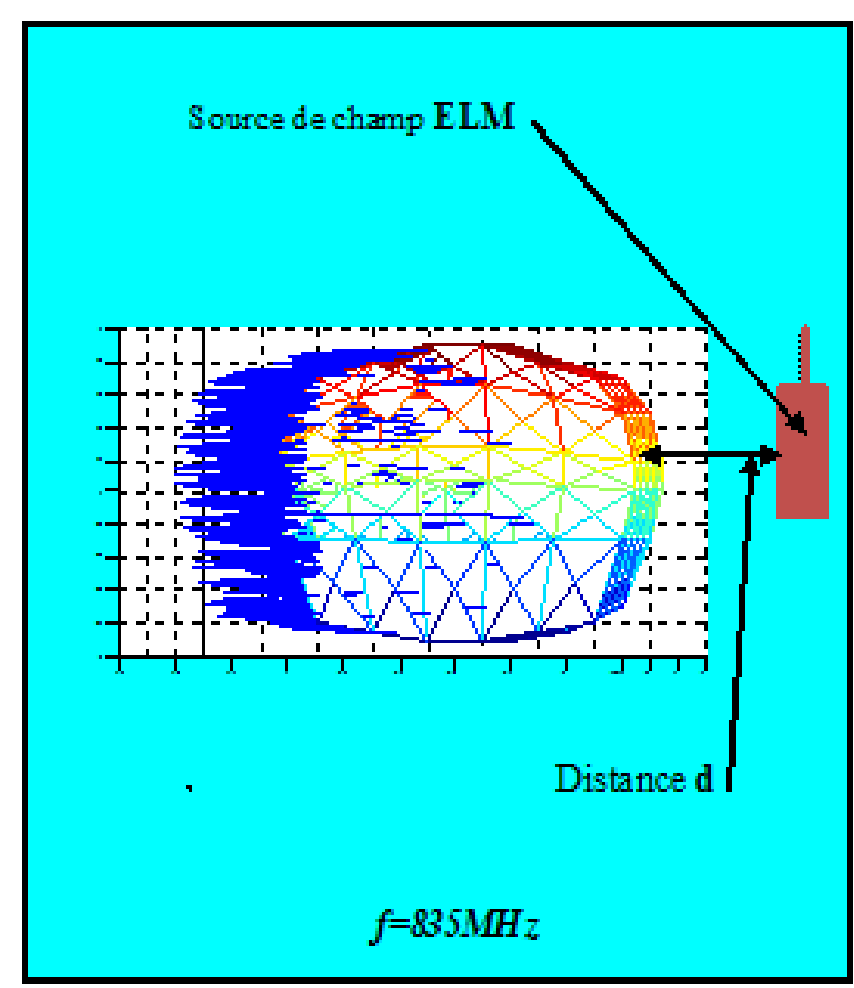

Figure 8: Distribution of magnetic field $\mathrm{H}$. Source close to the head $=0.3 \mathrm{~m}, \mathrm{f}=835 \mathrm{MHz}$ 


\section{International Journal of Science and Research (IJSR) \\ ISSN (Online): 2319-7064}

Index Copernicus Value (2013): 6.14 | Impact Factor (2014): 5.611

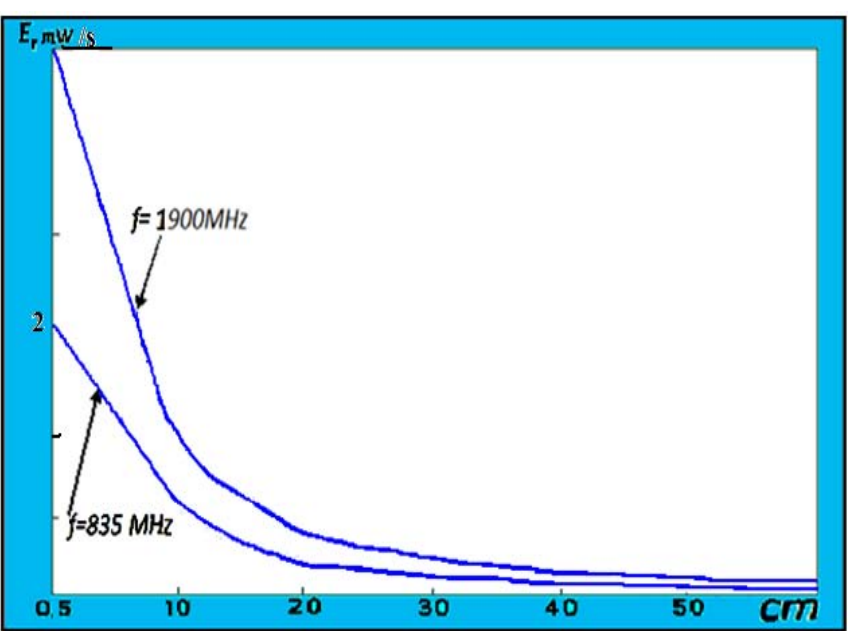

Figure 9: Energy variation $\operatorname{Er}(\mathrm{ELM})$ as a function of the distance $\mathrm{df}=835 \mathrm{MHz}$ and $\mathrm{f}=1900 \mathrm{MHz}$

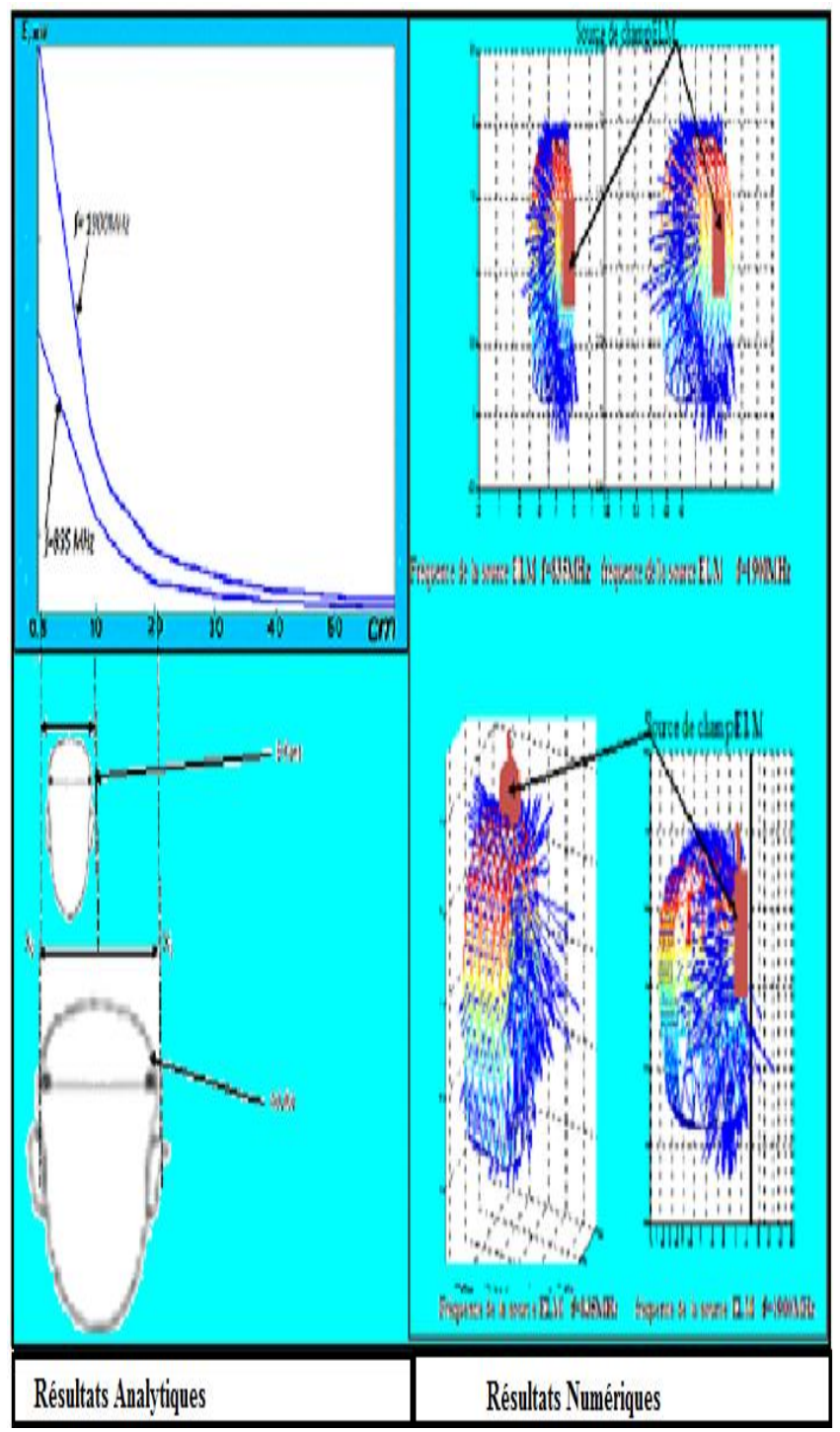

Figure 10: Comparison between analytic results and numerical resultsSource glued to thehead $\mathrm{f}=835 \mathrm{MHz}$ $\left(\sigma \mathrm{e}=0,45 \mathrm{~S} / \mathrm{m}, \varepsilon_{e}=16,40\right)$

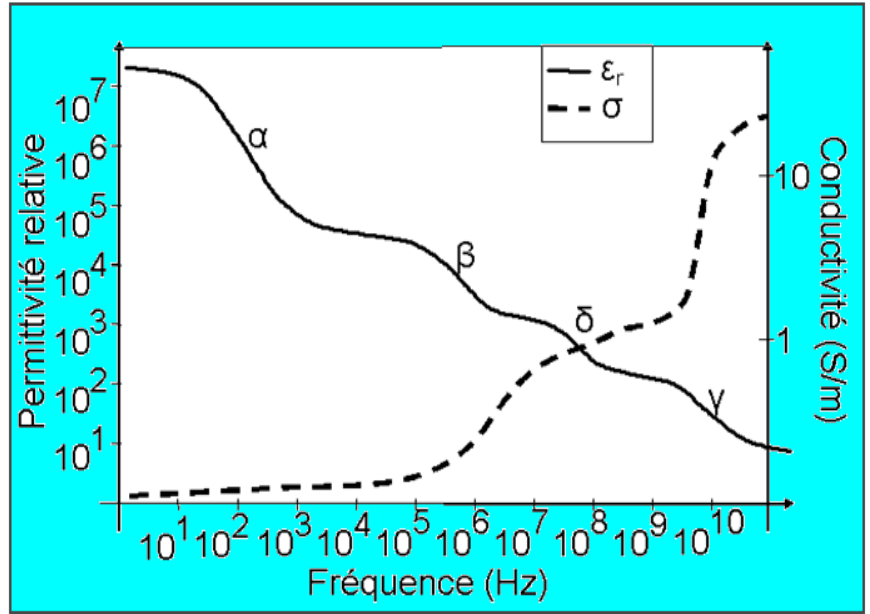

Figure 11: Variation of the $(\varepsilon, \sigma, \mu)$ as a function of the frequency $\mathrm{f}(\mathrm{Hz})$

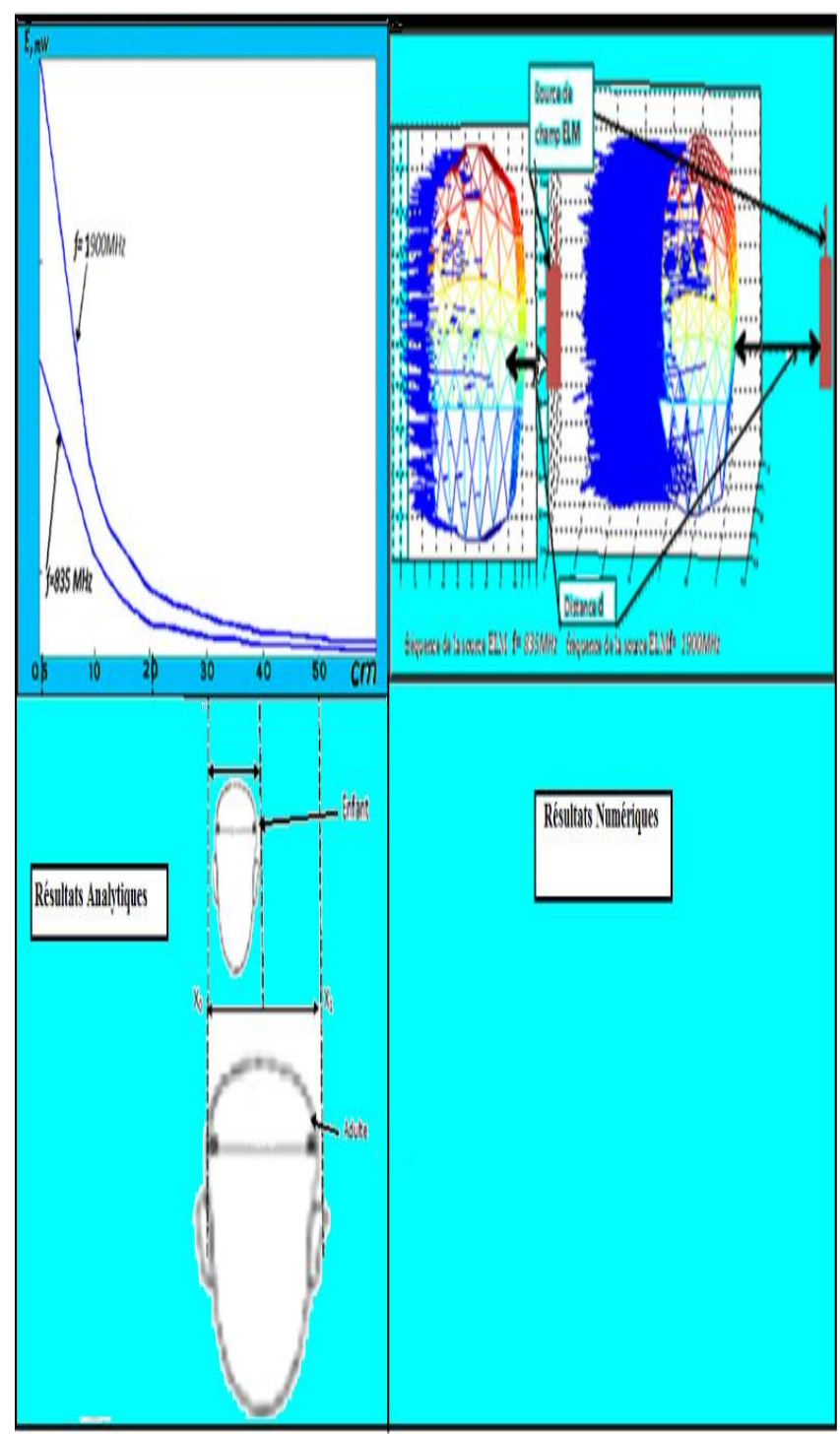

Figure 12: Comparison between analytical results and numerical results, Source far to the head,

$(\sigma \mathrm{e}=0,45 \mathrm{~S} / \mathrm{m}, \varepsilon \mathrm{e}=16,40)$

Analytical study confirmed the energy dissipated in the human head, was vary with the distance between the human head and the electromagnetic source, and with the variation of exterior medium parameters $(\varepsilon, \sigma, \mu)$.

Volume 5 Issue 1, January 2016 


\section{International Journal of Science and Research (IJSR) \\ ISSN (Online): 2319-7064}

Index Copernicus Value (2013): 6.14 | Impact Factor (2014): 5.611

Then this comparison between the two studies (analytical and numerical), gave us a confirmation of our numerical method for the selection of materials for good Protector of the human head against the electromagnetic fields.

\section{Conclusion}

In this article, we have some numerical results to assess the penetration of the electromagnetic field in the human head. We considered a source of radio frequency electromagnetic radiation (cell) in front of a human head at different distances. We carry a coupling between the integral method of external borders and the method of finite elements inside the human head to solve our Problem. We use the parameters of the external medium (the air in our case) $(\varepsilon, \sigma, \mu)$, which are in order the electrical permittivity, electrical conductivity and magnetic permeability.Numerical results show the contribution of each parameter of the external medium in the assessment of electromagnetic energy entering the human head, it will allow us to find the best protector for the human head based on the electromagnetic parameters $(\varepsilon, \sigma, \mu)$.

Hence they allow us to select the best material (external medium, protector), for a minimum of energy dissipated in the various points of our model (human head). Additionally, depending on the density of the source current, the geometry and the frequency of the radiating element. So it will lead us to a better biocompatibility in order to design several models of human head-mobile protecting interface

Numerical results (as arrows) showing the change in the values and the form of electromagnetic energy in each point inside the human head by varying every time a single parameter. Given the number of paramétrés, we cannot expose all the results in this document.

\section{References}

[1] N. Kuster, Schonborn F. 'Recommended minimum requirements, development of guidelines for exposure setups bio-experiments Addressing the health risk concern of wireless communications', Bio electromagnetics, Vol. 21, Issue 7, pp. 508-514, October 2000.

[2] F. Schonborn, Pokovic K., M. Burkhardt, N. Kuster 'Basis for optimization of in vitro exposure apparatus for health hazard evaluations of mobile communications' Bio electromagnetics, Vol. 22, Issue 8, pp. 547-559, November 2001.

[3] Wang Weidong, Yinghai Zhang Kaijie Zhou, Zhang Heng and Research on Asymmetric Characteristics of Mobile Communications System Based on Electromagnetic Radiation, Progress In Electromagnetics Research Symposium 2007, Prague, Czech Republic, August 27-30

[4] Andreas Christ, Marie-Christine Gosselin, Manuel Murbach, Sven Kühn, Zefferer Marcel, Katharina Honegger, Sonja Negovetic, and NielsKuster, Age Dependent Effects of RF Electromagnetic Fields on the basis ofRelevant Biological Parameters, Müchen, October 2007.

[5] Mr. Djennah, F. Rioux-Damidau, B. Bandelier "Calculation of electric charges and eddy currents with a formulation e" IEEE Transaction Magnetic Journal, 1997, Vol 32, pp 1322-1325

[6] Council Recommendation on the limitation of public exposure to electromagnetic fields $(0 \mathrm{~Hz}$ to $3000 \mathrm{GHz})$ $1999 / 519$ / EC, published in the Official Journal of the European Communities No 99 of 30 July 1999, pp. 0059. 0070, 12 July 1999 